\title{
Robust Face Tracking with Locally-Adaptive Correlation Filtering
}

\author{
Leopoldo N. Gaxiola ${ }^{1}$, Víctor Hugo Díaz-Ramírez ${ }^{1}$, Juan J. Tapia ${ }^{1}$, \\ Arnoldo Diaz-Ramirez ${ }^{2}$, and Vitaly Kober ${ }^{3}$ \\ 1 Instituto Politécnico Nacional - CITEDI, Ave. del Parque 1310, Mesa de Otay, \\ Tijuana B.C., 22510, Mexico \\ 2 Instituto Tecnologico de Mexicali, Mexicali B.C., 21396, Mexico \\ 3 Department of Computer Science, CICESE, Carretera Ensenada-Tijuana 3918, \\ Ensenada B.C., 22860, Mexico
}

\begin{abstract}
A face tracking algorithm based on locally-adaptive correlation filtering is proposed. The algorithm is capable to track a face with invariance to pose, gesticulations, occlusions and clutter. The target face is chosen at the beginning of the algorithm. Afterwards, a composite filter is designed to recognize the face in posterior frames. The filter is adapted online using information of current and past scene frames. The adaptive filter is constructed by combining several optimal templates designed for distortion invariant target recognition. Results obtained with the proposed algorithm using real-life scenes, are presented and compared with those obtained with a recent state-of-the-art tracking method, in terms of detection efficiency, tracking accuracy, and speed of processing.
\end{abstract}

\section{Introduction}

Face tracking is a widely investigated topic in computer vision. Video surveillance, human-computer interaction, and robotics are examples of face tracking applications. Face tracking consists in estimating the trajectory of a target's face while it moves through a detection zone [8 5 . A main difficulty in face tracking is that the observed scene is commonly degraded by additive noise, presence of a cluttering background, geometric modifications of the face such as pose and scaling, gesticulations, and nonuniform illumination. Additionally, eventual occlusions and real-time requirements are challenges that the tracking algorithm must solve.

Several approaches has been suggested to address target tracking 1312. Some algorithms require a strong a-priori knowledge of the target before tracking begin 3. Other algorithms only require a minimum knowledge of the target due the incorporation of online learning and adaptation mechanisms [127. Recently, the use of an adaptive correlation filtering for real-time target tracking has been suggested [1]. This method has shown that is competitive with standard tracking algorithms, but with much less complexity [412. In this approach, a correlation filter (template) is used to detect and locate the target within the observed scene in each frame. The template is updated online (adapted) accordingly with 
current and past scene observations, and by considering intraclass distortions. The correlation filter used in this approach is the Minimum Output Sum of Squared Error (MOSSE) filter [1].

The frequency response of the MOSSE filter is obtained by the minimization of the sum of squared error between two correlation planes. The first one is the correlation plane produced by the filter in response to the available views of the target. The second one is a desired correlation plane prespecified by the designer. The simulated plane is modeled as a Gaussian function $N(\mu, \boldsymbol{\Sigma})$, where its expected value $\mu=\left[\mu_{x}, \mu_{y}\right]^{T}$ is set at the expected coordinates of the target within the observed scene (location of the correlation peak), and $\boldsymbol{\Sigma}$ is the covariance matrix that defines the correlation peak width.

A main limitation of the MOSSE filter is that the actual location of the target in the scene cannot be known a-priori. For tracking applications, this issue is critical because the target is in constant motion and the expected location of the correlation peak cannot be accurately prespecified. Note that when this situation arises, a considerable bias may occur in the estimation of the location coordinates of the target 410. To avoid this shortcoming, we suggest the use of a locally adaptive composite filter synthesized as a combination of optimal filter templates designed for detection and location estimation of the target within the observed scene. First, a set of geometrically distorted versions of the reference image of the target is constructed. Afterwards, an optimal filter template is designed for each image, and therefore, the templates are combined to form a composite correlation filter. Note that by using the suggested approach, we avoid the need to specify the expected location of the target in the posterior frame. Therefore, the accuracy of target tracking is increased considerably in comparison with the MOSSE filter.

The paper is organized as follows. Section 2 explains the proposed filter design for target tracking. Section 3 describes the suggested algorithm for robust face tracking. Computer simulation results obtained with the proposed approach are presented and discussed in section 4 . The obtained results are compared with those obtained with the MOSSE filter in terms of detection efficiency, tracking accuracy, and speed of processing. Finally, section 5 presents our conclusions.

\section{Composite Filter Design Using Optimal Templates}

We are interested in the design of a correlation filter able to recognize a face image when it is embedded into a disjoint background, and the scene is corrupted with additive noise. Also, the filter needs to be capable to recognize geometrically distorted versions of the target, such as rotations and scaling. Let $T=\left\{t_{i}(x, y) ; i=1, \ldots, N\right\}$ be an image set containing geometrically distorted versions of the target to be recognized. The input scene is assumed to be composed by the target $t(x, y)$ embedded into a disjoint background $b(x, y)$ at unknown coordinates $\left(\tau_{x}, \tau_{y}\right)$, and the whole scene is corrupted with additive noise $n(x, y)$, as follows:

$$
f(x, y)=t\left(x-\tau_{x}, y-\tau_{y}\right)+b(x, y) \bar{w}\left(x-\tau_{x}, y-\tau_{y}\right)+n(x, y),
$$




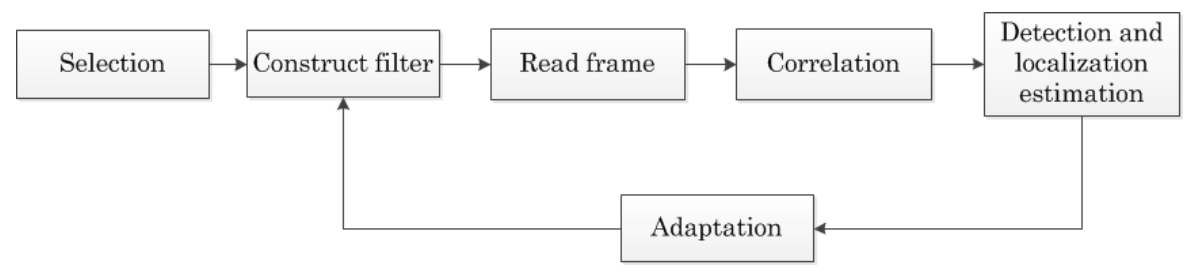

Fig. 1. Basic diagram of the proposed face tracking system

where $\bar{w}(x, y)$ is a binary function defined as zero inside the target area, and unity elsewhere. The optimum filter for detecting the target from Eq. (1), in terms of the signal to noise ratio (SNR) [1] and the minimum variance of measurements of location errors (LE) [9], is the generalized matched filter (GMF) 96., whose frequency response is given by

$$
H^{*}(u, v)=\frac{T(u, v)+\mu_{b} \bar{W}(u, v)}{P_{b}(u, v) \otimes|\bar{W}(u, v)|^{2}+P_{n}(u, v)} .
$$

In Eq. (2), $T(u, v)$ and $\bar{W}(u, v)$ are the Fourier transforms of $t(x, y)$ and $\bar{w}(x, y)$, respectively; $\mu_{b}$ is the mean value of the background $b(x, y)$; and $P_{b}(u, v)$ and $P_{n}(u, v)$ denote power spectral densities of $b_{0}(x, y)=b(x, y)-\mu_{b}$ and $n(x, y)$, respectively. The symbol " $\otimes$ " denotes convolution.

Let $h_{i}(x, y)$ be the impulse response of a GMF constructed for the $i$ th available view of the target $t_{i}(x, y)$ in $T$. Let $H=\left\{h_{i}(x, y) ; i=1, \ldots, N\right\}$ be the set of all GMF impulse responses constructed for all training images $t_{i}(x, y)$. Additionally, let $S=\left\{s_{i}(x, y) ; i=1, \ldots, M\right\}$ be an image set containing $M$ unwanted patterns to be rejected. We want to synthesize a filter capable to recognize all target views in $T$ and reject the false patterns in $S$, by combining the optimal filter templates contained in $H$, and by using only a single correlation operation. The required filter $p(x, y)$, can be constructed as follows:

$$
p(x, y)=\sum_{i=1}^{N} \alpha_{i} h_{i}(x, y)+\sum_{i=N+1}^{N+M} \alpha_{i} s_{i}(x, y)
$$

where the coefficients $\left\{\alpha_{i} ; i=1, \ldots, N+M\right\}$ are chosen to satisfy prespecified output values for each pattern in $U=T \cup S$. Using vector-matrix notation, we denote by $\mathbf{R}$ a matrix with $N+M$ columns, where each column is the vector version of each element of $U$. Let $\mathbf{a}=\left[\alpha_{i} ; i=1, \ldots, N+M\right]^{T}$ be a vector of coefficients. Thus, Eq. (3) can be rewritten as

$$
\mathbf{p}=\mathbf{R a}
$$

Let us denote by

$$
\mathbf{u}=[\underbrace{1, \ldots, 1}_{N \text { ones }}, \underbrace{0, \ldots, 0}_{M \text { zeros }}]^{T}
$$


the desired responses to the training patterns, and denote by $\mathbf{Q}$ the matrix whose columns are the elements of $U$. The response constraints can be expressed as

$$
\mathbf{u}=\mathbf{Q}^{+} \mathbf{p},
$$

where superscript "+" denotes complex conjugate. Substituting Eq. (4) into Eq. (6), we obtain

$$
\mathbf{u}=\mathbf{Q}^{+} \mathbf{R} \mathbf{a} .
$$

Thus, the solution for $\mathbf{a}$, is

$$
\mathbf{a}=\left[\mathbf{Q}^{+} \mathbf{R}\right]^{-1} \mathbf{u} .
$$

Finally, substituting Eq. (8) into Eq. (4), the solution for the composite filter is given by

$$
\mathbf{p}=\mathbf{R}\left[\mathbf{Q}^{+} \mathbf{R}\right]^{-1} \mathbf{u} .
$$

Note that the value of the correlation peak when using the filter given in Eq. (9), is expected to be close to unity for true-class objects, and close to zero for false-class objects.

\section{Face Tracking with Locally-Adaptive Correlation Filtering}

In this section we describe the proposed algorithm for robust face tracking based on adaptive correlation filtering. The algorithm needs to be robust to gesticulations, pose changes, scaling, and occlusions. The algorithm starts with an initialization step in where the target face is defined. Next, the algorithm generates several geometrically distorted versions of the face image and construct the sets $T$ and $H$. Actually, if a-priori information of unwanted patterns to be rejected is available, such as other faces or background fragments, we can use it to construct the set S. Afterwards, a composite filter is synthesized using Eq. (9). The next step is to correlate the composite filter with an updated tracking window (taken from a new frame), and calculate the discrimination capability (DC) of the resultant correlation plane. The DC is a measure of the ability of the filter to distinguish a target from unwanted objects; it is defined by [9]

$$
D C=1-\frac{\left|c^{b}\right|^{2}}{\left|c^{t}\right|^{2}},
$$

where $c^{b}$ is the value of the maximum correlation sidelobe in background area and $c^{t}$ is the value of the correlation peak generated by the target. A DC value close to unity indicates that the filter has a good capability to distinguish between the target and any false object. Negatives values of the DC indicate that the filter is unable to detect the target. Next, if the obtained DC is greater than a prespecified threshold $\left(D C>D C_{t h}\right)$, then the target is considered as detected and the composite filter is updated using as new reference image the detected face in the current frame. Otherwise, the target is rejected. It can be seen, that the composite filter is updated online for each frame without the need of any offline supervised training process [3]. The operation of the algorithm is depicted in Fig. 1] and their steps are summarized in Algorithm 1. 
Algorithm 1. Proposed algorithm for robust face tracking.

STEP 1 Read a scene frame $f_{k}(x, y)$ from the video sequence and chose a target face from a small window $b_{k}(x, y)$.

STEP 2 Create geometrically distorted versions of $b_{k}(x, y)$ and construct the sets $T$ and $H$.

STEP 3 Synthesize a composite filter $p_{k}(x, y)$ (using Eq. (9)) trained for sets $T$ and $H$ and construct an adaptive filter as follows:

$$
p_{k}(x, y)=\alpha p_{k}(x, y)+(1-\alpha) p_{k-1}(x, y),
$$

where $\alpha$ is a constant, and $p_{k-1}(x, y)$ is the filter for past frame. Note that $p_{k-1}(x, y)=0$ when $k=1$.

STEP 4 Compute the cross-correlation function $c_{k}(x, y)$ between the adaptive filter $p_{k}(x, y)$ and the window $b_{k}(x, y)$. Next, calculate the DC using Eq. (10).

STEP 5 If $D C>D C_{t h}$, estimate the current location of the target as $\left(\tau_{x}, \tau_{y}\right)=$ $\operatorname{argmax}\left\{\left|c_{k}(x, y)^{2}\right|\right\}$. Otherwise, read a new frame and update the window $x, y$

$b_{k}(x, y)$ by placing its origin at the current estimate of the location of the target. Next go to step 2.

\section{Results}

The proposed algorithm is tested using a real-life video sequence that contains pose changes, scaling, blurring and occlusions. The algorithm is compared with the algorithm suggested by Bolme et. al [1] which is based on the MOSSE filter. Both algorithms are evaluated in terms of detection efficiency, tracking accuracy, and speed of processing. Detection efficiency is characterized by the DC. Tracking accuracy is evaluated in terms of tracking errors, and speed of processing is given by the frames-per-second (fps) that the tracking algorithms are able to process. The test sequence contains 2250 monochrome scene frames, each of them with $640 \mathrm{x} 480$ pixels. The signal range is $[0,1]$ with 256 quantization levels. The sets $T$ and $H$ used for the filter synthesis are constructed as follows: for a given target image four different rotated versions of the target are generated within an interval of \pm 10 degrees; using an increment step of 5 degrees.

Fig. 2] shows examples of scene frames of the test sequence. The green square identifies the proposed tracker, whereas the red square identifies the MOSSE tracker. Observe that the proposed algorithm is able to track the target in all frames, even in the presence of pose changes, scaling, blurring, and occlusions. Instead, the MOSSE tracker loses the target in several frames (see Fig. 2(c), (g) and (i)). In addition, note that the MOSSE tracker yields a considerable bias in the location estimation of the target when the face suffer geometrical changes (see Fig. 2 (b), (f), and (h)).

Fig. 3 shows the obtained DC of both tested algorithms for all frames of the test sequence. The circles correspond to the DC values of the trackers for the scene frames shown in Fig. 2. When the proposed algorithm is used only 


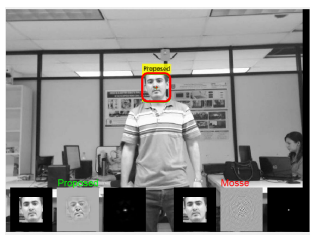

(a)

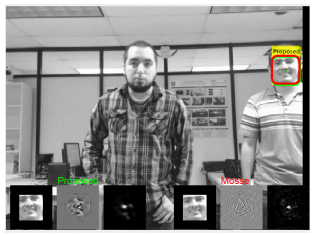

(d)

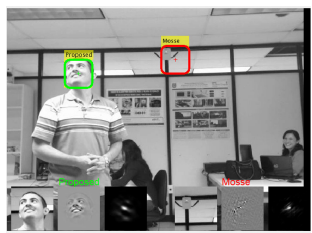

(g)

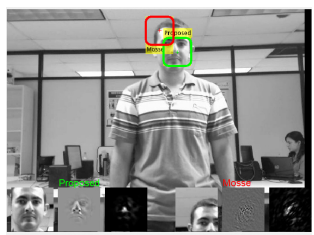

(b)

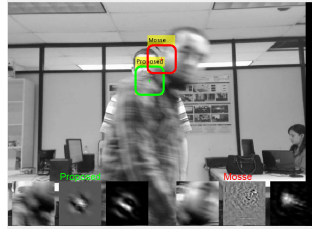

(e)

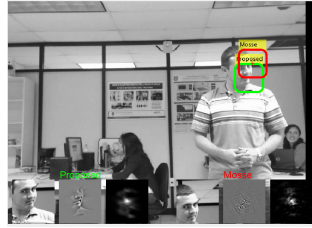

(h)

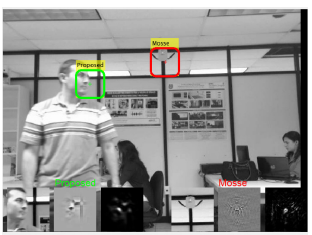

(c)

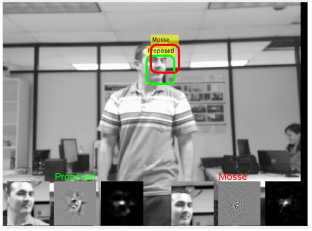

(f)

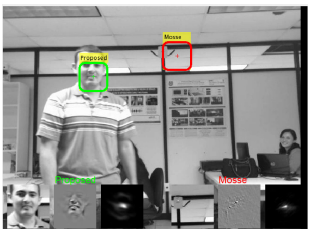

(i)

Fig. 2. Examples of scene frames in the video test sequence. (a) Target face (reference image), (b) scaling, (c) out-of-plane rotation, (d) close to edge, (e) occlusion, (f) blurring, (g)-(i) pose changes.

few frames yield DC values below $D C_{t h}=0.2$. This means that the target is not detected. Observe Fig. 2(e) in where the target is occluded. The DC value obtained for this frame is below $D C_{t h}$. So, the algorithm handles the occlusion by stopping the update of the filter while $D C<D C_{t h}$ (STEP 5 of Algorithm 11). The MOSSE tracker yields a DC value above $D C_{t h}$ for the frame of Fig. 2(e). In this case, the MOSSE filter detects the false target and the algorithm tracks him. According to Fig. 3 the proposed tracker yields a better performance in terms of the DC than the MOSSE tracker. This means the the proposed composite filter yields a better recognition performance than the MOSSE filter for target tracking. Also, Fig. 3 shows the scene frames in where a tracking algorithm loses the target. This is identified by a red ellipse. Note that the proposed algorithm is able to detect and track the target in all frames, whereas the MOSSE algorithm loses the target in four segments of the video sequence. Examples of this situations are depicted in Fig. 2(c), (g) and (i). 


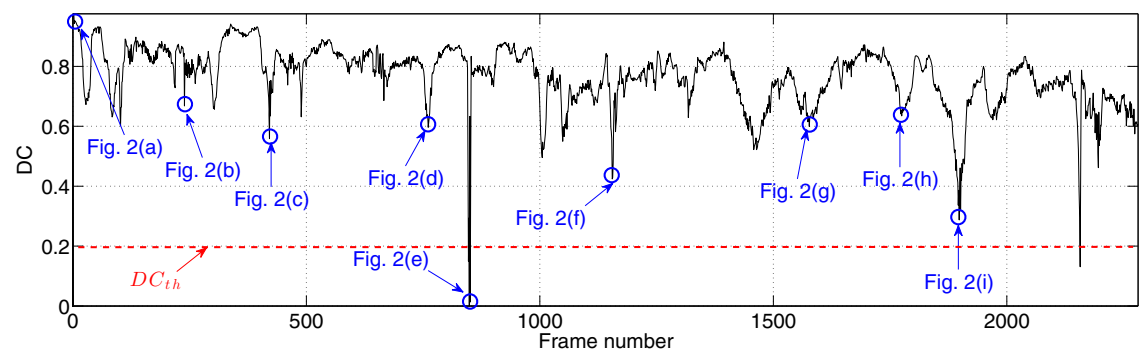

(a)

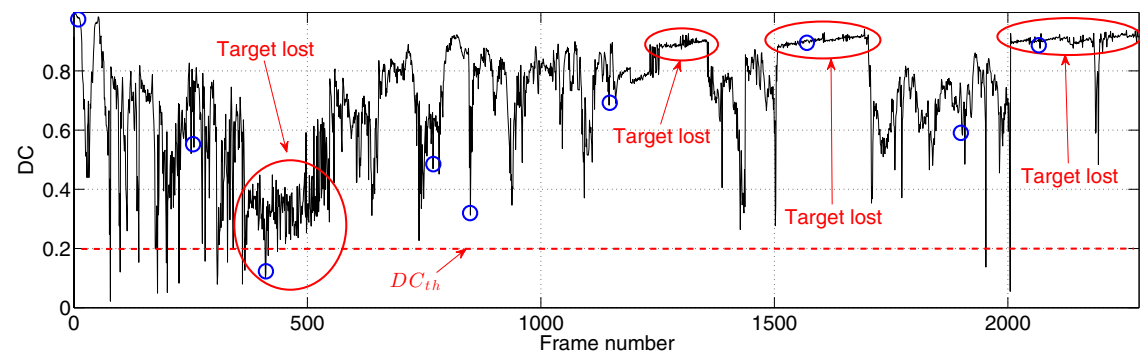

(b)

Fig. 3. DC performance of tracking algorithms when processing 2250 scene frames. (a) Proposed tracker, (b) MOSSE tracker.

The algorithms were implemented in MATLAB on a $3.4 \mathrm{Ghz}$ Intel-i7 desktop computer with 16 GB of RAM memory, running on Windows 7 64-bits operating system. The MOSSE and proposed algorithms processed 2250 frames at the speed of $22.1 \mathrm{fps}$ and $20.5 \mathrm{fps}$, respectively. Let $P$ be the time that takes an algorithm to process a video sequence of duration $T$. The real-time factor [2] is defined as $R T=\frac{P}{T}$, where a value of $R T \leq 1$ indicates that the algorithm operates in real-time. Table 1 shows the performance of the proposed and MOSSE algorithms in terms of fps and the $R T$. We see that the MOSSE algorithm is a little bit faster than the proposed algorithm. This is because the suggested composite filter is synthesized in a two-step procedure. However, this difference is negligible when considering the great improvement in face tracking that is obtained when using the proposed filter.

Table 1. Speed of processing of proposed and MOSSE tracking algorithms

\begin{tabular}{cc}
\cline { 2 - 2 } & \multicolumn{2}{c}{ Real-time factor } \\
fps & $R T=\frac{P}{T}$ \\
\hline \hline Proposed 20.5 & 1.46 \\
MOSSE 22.1 & 1.35 \\
\hline
\end{tabular}




\section{Conclusions}

A locally-adaptive algorithm for robust face tracking was presented. The algorithm detects and locates a face in each frame of a video sequence using a composite correlation filter that is adapted online. The filter is constructed by combination of optimal filter templates designed for target detection and location estimation. The proposed filter was compared with the MOSSE filter for face tracking in a real-life video sequence. Computer simulation results showed that the proposed filter is significantly more robust and accurate than the MOSSE filter, in the presence of pose changes, scaling, and occlusions. The real-time performance of the tested filters was evaluated using MATLAB. We found that the synthesis of the MOSSE filter is slightly faster than the synthesis of the proposed filter. However, this latency is not significant compared with the improvement of face tracking that is obtained with the proposed algorithm.

\section{References}

1. Bolme, D., Beveridge, J., Draper, B., Lui, Y.M.: Visual object tracking using adaptive correlation filters. In: IEEE Conference on Computer Vision and Pattern Recognition (CVPR), pp. 2544-2550 (2010)

2. Butazzo, G.: Hard Real-Time Computing Systems: Predictable Scheduling Algorithms and Applications, 3rd edn. Springer (2011)

3. Diaz-Ramirez, V.H., Picos, K., Kober, V.: Target tracking in nonuniform illumination conditions using locally adaptive correlation filters. Opt. Commun. 323, 32-43 (2014)

4. Henriques, J.F., Caseiro, R., Martins, P., Batista, J.: Exploiting the circulant structure of tracking-by-detection with kernels. In: Fitzgibbon, A., Lazebnik, S., Perona, P., Sato, Y., Schmid, C. (eds.) ECCV 2012, Part IV. LNCS, vol. 7575, pp. 702-715. Springer, Heidelberg (2012)

5. Huang, C., Ding, X., Fang, C.: Pose robust face tracking by combining view-based AAMs and temporal filters. Comput. Vis. Image Underst. 116(7), 777-792 (2012)

6. Javidi, B., Wu, J.: Design of filters to detect a noisy target in nonoverlapping background noise. J. Opt. Soc. Am. A 11(10), 2604-2612 (1994)

7. Kalal, Z., Mikolajczyk, K., Matas, J.: Tracking-learning-detection. IEEE Trans. Pattern Anal. Mach. Intell. 34(7), 1409-1422 (2012)

8. Kim, M., Kumar, S., Pavlovic, V., Rowley, H.: Face tracking and recognition with visual constraints in real-world videos. In: IEEE conference on Computer Vision and Pattern Recognition (CVPR), pp. 1-8 (2008)

9. Kober, V., Campos, J.: Accuracy of location measurement of a noisy target in a nonoverlapping background. J. Opt. Soc. Am. A 13(8), 1653-1666 (1996)

10. Lubing, Z., Han, W.: Facial landmark localization via boosted and adaptive filters. In: IEEE International Conference on Image Processing (ICIP), pp. 519-523 (2013)

11. Vijaya-Kumar, B.V.K., Hassebrook, L.: Performance measures for correlation filters. Appl. Opt. 29(20), 2997-3006 (1990)

12. Wu, Y., Lim, J., Yang, M.: Online object tracking: A benchmark. In: IEEE Conference on Computer Vision and Pattern Recognition (CVPR), pp. 2411-2418 (2013)

13. Yilmaz, A., Javed, O., Shah, M.: Object tracking: A survey. ACM Comput. Surv. 38(4) (2006) 